\title{
Femur Stress - Can You Take It?
}

\section{Saagar Patel, MD*, Areeb Masood, MD, Girija Rajakumar, MD, Pritish Bawa, MD, Haitham Awdeh, MD and Manickam Kumaravel, MD}

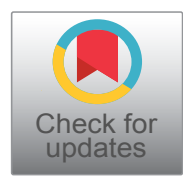

\author{
University of Texas Health Science Center at Houston, McGovern Medical School, USA
}

*Corresponding author: Saagar Patel, University of Texas Health Science Center at Houston McGovern Medical School, 6431 Fannin St Houston, TX 77030, USA, Tel: 713-500-7702

\begin{abstract}
Background and objective: Outline the biomechanics and pathophysiology of stress-induced injuries to the femur. Provide various imaging presentations of stress injuries of the femur including radiographs, computed tomography (CT), and Magnetic resonance imaging (MRI). Understand treatment modalities, recognize complications and become proficient in avoiding pitfalls.

Methods: This paper will discuss detailed macro and microanatomical features of the femur and associated soft tissues such as muscles, tendons, and cartilage. A review of biomechanics of the femur with consideration to the remodeling process of the femur will be provided. Emphasis will be placed on structural integrity and composure of trabecular bone and its response to stress.

Results: Multiple cases of stress response and fractures in the femur using various modalities such as radiographs, $\mathrm{CT}$, and MRI will be used to review presentations of stress related injuries to the femur. Treatment options and imaging follow up for these cases will also be discussed.
\end{abstract}

Conclusion: The learner will be exposed to key concepts of stress related injuries and informed of pitfalls.

\section{Keywords}

Radiology, Femur fracture, Femur stress, Imaging review

\section{Introduction}

The femur is a crucial anatomical and physiological structure that allows for humans to ambulate and maintain balance upright with ease. Similar to other long bones, the femur is subject to remodeling and turnover with variability based on use and trauma. It has high compressive and tensile strength and is the attachment point for major muscle groups such as the iliopsoas, gluteal, quadriceps, and hamstrings. With multi-directional forces acting on the long bone, the femur becomes prone to stress-related injuries including microfractures. Incidence of femoral stress fractures is not entirely established, displaying variability among athletes and military trainees. Of all sports and military associated stress fractures, $7.2 \%$ were located in the femur [1]. Stress fractures, if left untreated, can progress to a more complex and comminuted fracture, thus furthering injury, creating functional deficits, and worsening long term prognosis. Understanding the anatomy, biomechanics, and pathophysiology of femur stress fractures can increase diagnostic accuracy and improve prognosis for patients.

\section{Anatomy}

The proximal femur, part of the hip joint is a balland-socket joint that allows for abduction, adduction, extension, flexion, external rotation and internal rotation of the lower leg. The medial side of the femur is bordered by the adductor muscles, which originate at the inferior portion of the acetabulum and pubis. The middle and distal posterior third portions of the femoral shaft provides adductor muscles insertion. Hip flexion is mainly controlled by the iliopsoas muscle group, namely psoas major and iliacus that inserts on the lesser trochanter of the femur. Hip external rotators such as the gluteus medius and minimus attach to the greater trochanter of the femur. The vastus medialis and lateralis muscles attaches on the lateral aspect of the femur. This vastus muscle group extends to the lower leg and distributes forces/weight throughout the femur to the femoral head, which is adjacent to the acetabulum Figure 1.

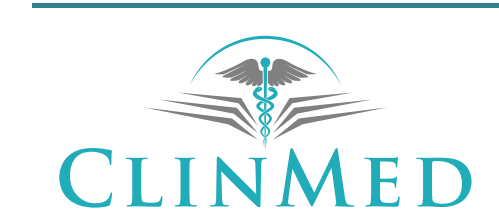

INTERNATIONAL LIBRARY
Citation: Patel S, Masood A, Rajakumar G, Bawa P, Awdeh H, et al. (2019) Femur Stress - Can You Take It?. Int Arch Orthop Surg 2:014. doi.org/10.23937/2643-4016/1710014

Accepted: October 05, 2019; Published: October 07, 2019

Copyright: (c) 2019 Patel S, et al. This is an open-access article distributed under the terms of the Creative Commons Attribution License, which permits unrestricted use, distribution, and reproduction in any medium, provided the original author and source are credited. 


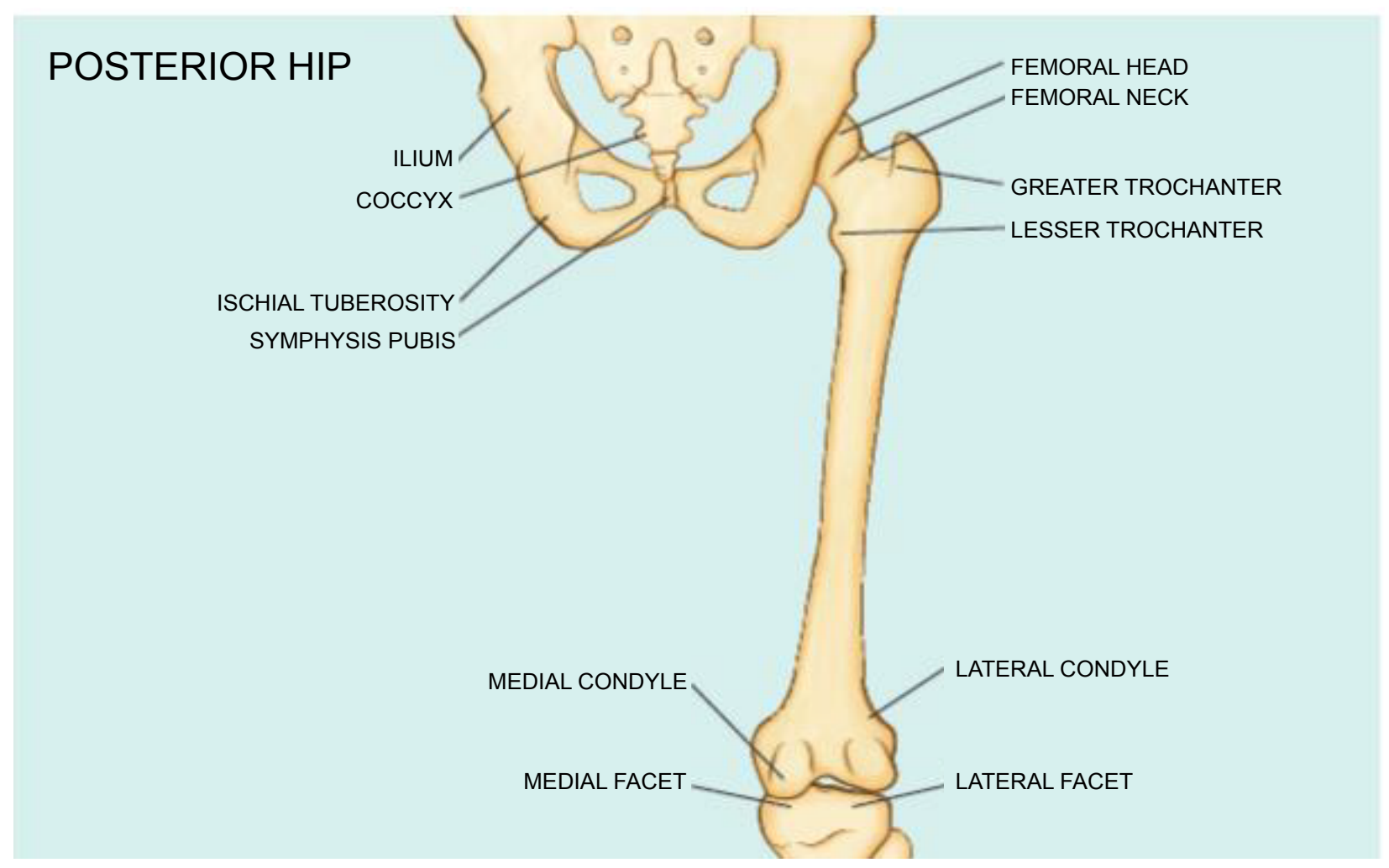

Figure 1: Anatomy of posterior hip outlining major anatomical landmarks.

The femoral head has multidirectional trabecula to assist in distributing forces and reinforce the femoral neck. There are groups of trabecular tissue at the proximal femur, all of which assist in supporting tensile and compressive forces. The growth and proliferation of trabecular tissue in remodeled bone is dependent on the location of maximum forces and stress lines, often referred to as Wolf's law [2]. Therefore, the convergence of the trabecular tissue and convergence of increased forces at the femoral neck is associated with an increased predisposition for fractures. There are two main trabecular patterns at the femoral neck: The principal tensile trabeculae and principal compressive trabeculae. The principal tensile trabeculae form a band between the lateral aspect of the greater trochanter to the fovea. The principal compressive trabeculae band extends vertically from the medial aspect of the femoral head to the femoral neck [3]. The increased stress and load at this convergence point (known as Ward's Triangle) can lead to increased bone turnover, especially compared to distal aspect of the femur [4]. Failure of appropriate trabecular formation can also predispose patients to injury, especially stress injury or fracture.

The distal aspect of the femur consists of ligamentous attachments of the knee including the anterior cruciate ligament $(A C L)$, posterior cruciate ligament $(P C L)$, medial collateral ligament (MCL), and lateral collateral ligament (LCL). It also includes the medial and lateral condyle with an articulating surface with the tibia and patella. Distal articulation with meniscal involvement is crucial to the femur's weight distribution and stress management.

\section{Etiology of stress fractures}

Long bones are subjected to two types of pathophysiologic forces that result in stress fractures. One is abnormal loading on normal bone, and the other is normal load on an abnormal bone. The first type (abnormal load on normal bone) is a consequence of excessive use and force and referred to as "fatigue fractures". The current theory is that overuse and increased frequency of weight bearing activity causes an imbalance between the osteoblastic and osteoclastic cells. Turnover of the cortical matrix is increased, especially in heavy load bearing areas such as the femoral neck, Ward's triangle, and subtrochanteric cortex. The increased osteoclastic function causes a weakening of the bone matrix leading to a pathological fatigue stress fracture. Patients are often seen to have thickened trabecular formation along the medial aspect of the femoral neck, secondary to the overall increase in extrinsic stress or weight bearing activities. This thickening can be notated as an osteophyte formation and can be a clue as to underlying improper bone remodeling. The efficiency in bone remodeling is greatly dependent on the vascularity of the cortical and subchondral bone [5].

The second type (normal load on abnormal bone) is due to metabolic and/or hormonal imbalance. This leads to improper osteoid formation and decreased strength of the cortical bone, resulting in "insufficiency 
fractures". Additionally, imbalance of electrolytes, hormones, and nutrition can cause a stress fracture even during normal weight bearing activity. There is an increased propensity for stress fractures to occur in long bones when the cause is secondary to metabolic insufficiency [6].

In femurs, stress fractures most commonly occur at the medial aspect, at the junction of the proximal and middle third of femoral shaft [7]. The increased propensity of medial third stress fractures are secondary to the antero-lateral bend of the femur. The associated adductor complex increases the compression forces and fatigue on the medial side.

\section{Types of stress fractures in the femur}

Based on etiology two types of stress fractures, tensile and compressive, can present subtly with similar clinical symptoms on plain radiographs. It is believed that compressive fractures generally occur in younger patients, while "transverse" or tensile fractures occur in an older population. Elderly women have an increased risk for tensile stress fracture secondary to osteogenic long bones and decreased mineralization of the cortical matrix [8]. If unrecognized or untreated, these fractures at the femoral neck can lead to two serious complications: valgus and varus compaction fracture.

Due to the normal convexity of the femur, there is an increased tensile force on the lateral aspect of the femur often leading to a transverse cortical stress fracture in predisposed individuals. Athletes may ex- perience excessive fatigue of specific muscle groups which leads to an imbalance between the forces acting on the long bones of the femur and results in a typical tensile stress fracture. Typical tensile stress fractures of the femoral neck are seen at the "midpoint of the lateral aspect of the femoral neck" and have a high risk of complications. Atypical tensile stress fractures are defined by cortical disruptions on the proximal or distal third of femoral shaft. Recent studies have indicated that "typical" stress fractures in the lateral femoral neck likely alters the treatment plan (surgical vs. non-surgical) [9].

Unlike tensile stress fractures, compressive stress fractures occur on the medial aspect of the femur. The adductors are medially attached to the proximal and distal aspects of the femur exacerbating the convexity of the femur. In patients with excessive fatigue, weight, or metabolic insufficiency, the bone becomes impacted. This leads to stress injuries and/or microfracture, furthering to a stress fracture.

\section{Varus and valgus impaction factures}

Unrecognized stress fractures of the femoral neck can progress into a varus or valgus deformities, often referred to as impaction fracture. A valgus deformity can result from a tensile "typical" stress fracture at the femoral neck. A varus deformity can result from a compressive stress fracture at the femoral neck. Bone remodeling process is disrupted or misaligned, causing a need for surgical fixation or correction.

The femur articulates proximally with the acetabu-

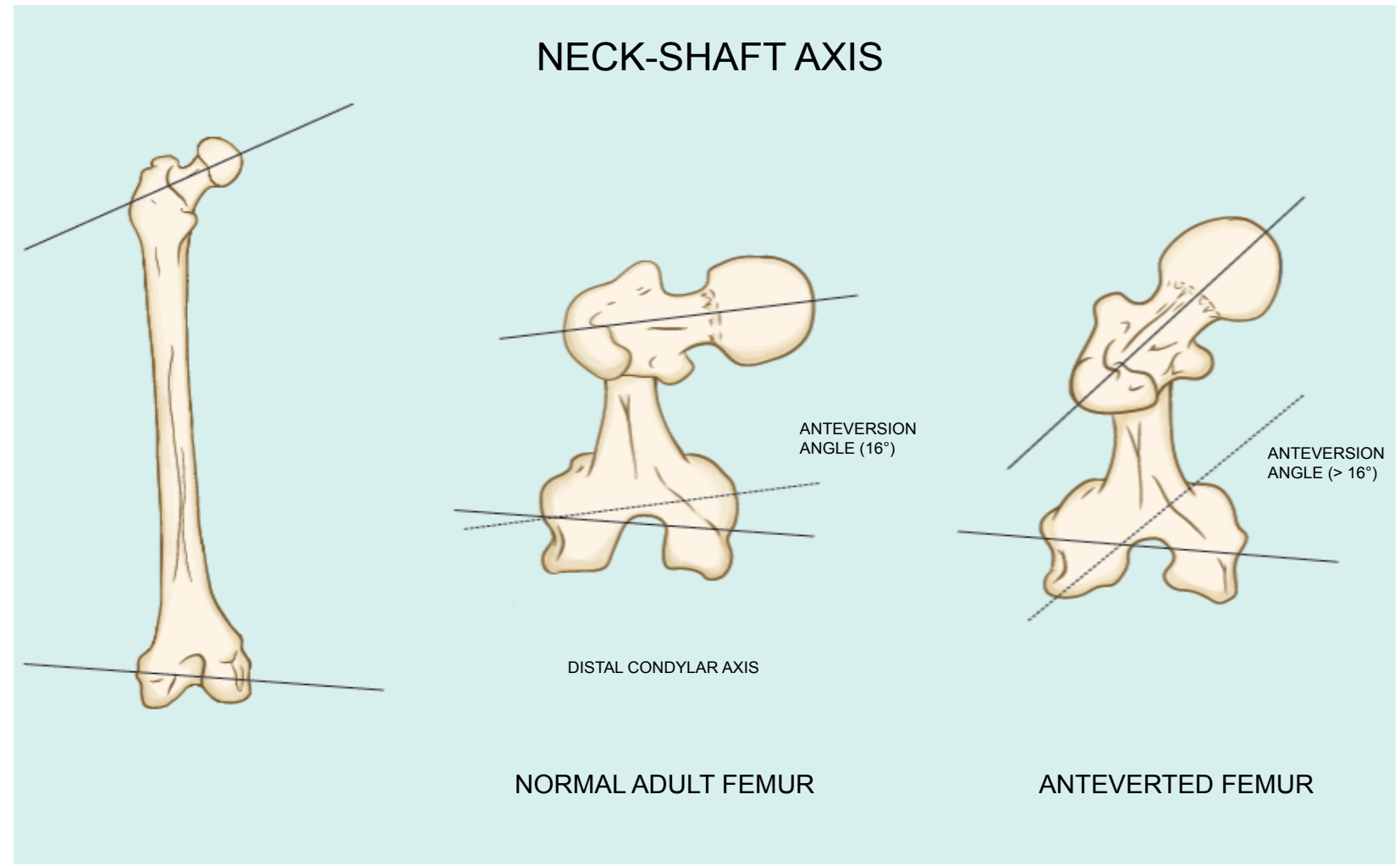

Figure 2: Anatomy of the adult femur indicating normal distal condylar axis (16 degrees) and anteverted femur ( $>16$ degrees). 


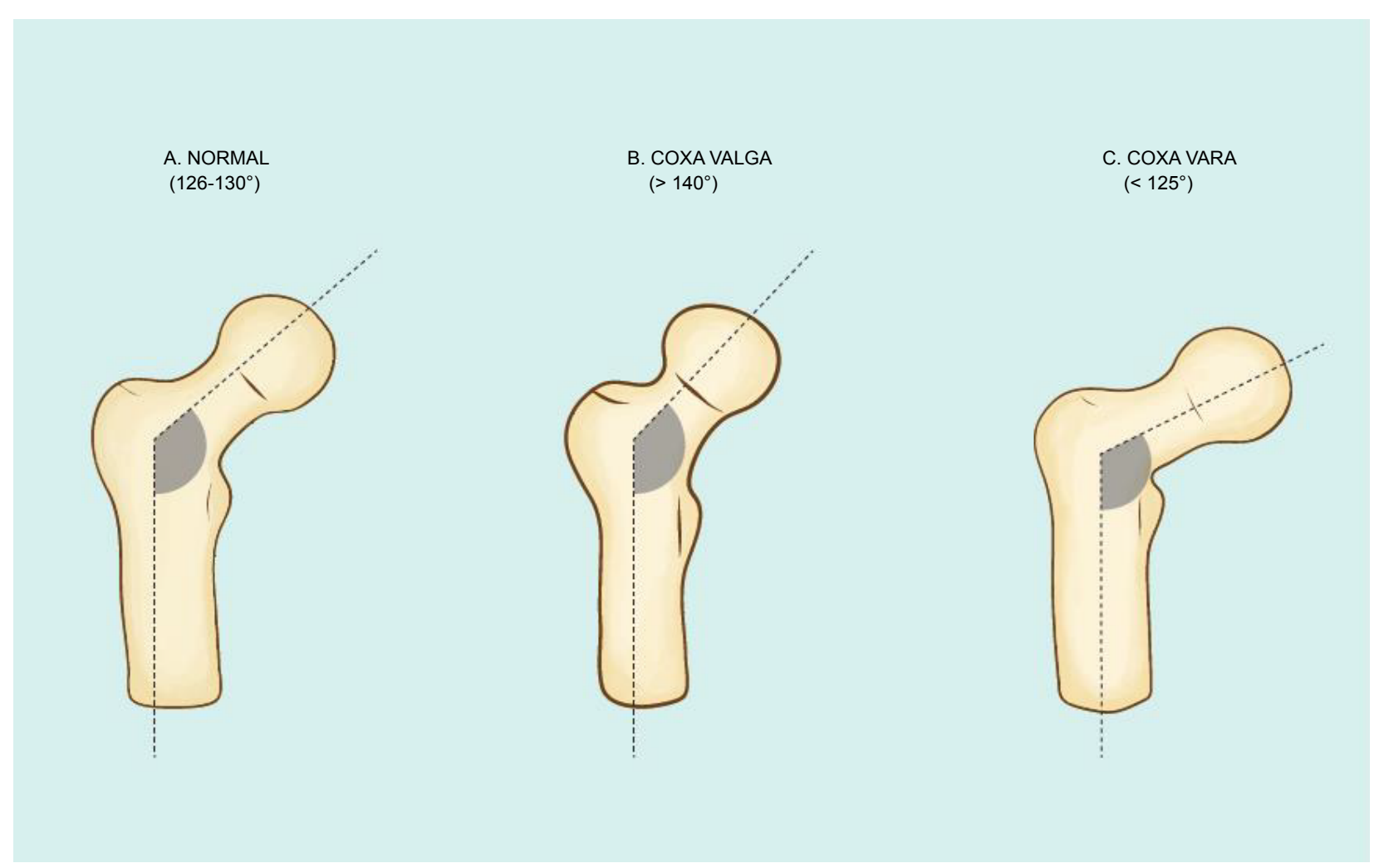

Figure 3: Femoral shaft and femoral neck angle with variation depicted as Coxa Valgus and Coxa Varus.

Table 1: Garden's classification of femoral neck fractures [10].

\begin{tabular}{|l|l|l|}
\hline Type & Description & Displacement \\
\hline I & Incomplete fracture, valgus impacted & Non-displaced \\
II & Complete fracture & Non-displaced \\
III & $\begin{array}{l}\text { Complete fracture, partial } \\
\text { displacement }\end{array}$ & Displaced \\
IV & $\begin{array}{l}\text { Complete fracture, fully displaced } \\
\text { Displaced }\end{array}$ \\
\hline
\end{tabular}

lum and distally with the tibia at specific angles to allow for appropriate rotation and distribution of forces. The normal femoral neck anteversion is 15 degrees. This angle is measured along the femoral neck and head relative to the articulation of the femur to the tibia. The angle of the femoral head to the femoral shaft is normally around 126-139 degrees which is essential for proper muscle attachment and force distribution among adductor muscles (Figure 2 and Figure 3).

There are multiple grading schemes for femoral head and neck fractures, one of them being the Garden's classification (Table 1).

Valgus impaction fractures is a complication of stress fracture and are generally associated with the femoral neck and head, and usually happens due to excessive training, running or falling. A valgus fracture indicates a shift of the femoral head superiorly, increasing the femoral head angle greater than 135 degrees. The fracture line can be subtle and often mistaken for an osteophyte or subclinical cortical reaction. Valgus impaction fractures are indicated by subtle displacement on MRI imaging with associated

\section{osteoedema.}

A valgus fracture would be classified as a Garden I or II [11]. Valgus stress fractures are less likely to displace or cause disruption to adjacent structures.

Varus impaction fracture is similar to valgus, but the femoral head is displaced inferiorly leading to an angle less than 120 degrees. Complications such as osteonecrosis are more common in varus impaction fractures. Such complications usually require surgical intervention. The femoral head and neck are highly perfused by arterial trunks that branch off the femoral artery, which runs inferiorly to the neck and lesser trochanter. A varus fracture with displacement can impinge and disrupt the blood flow to the capsule, leading to rapid osteonecrosis if not addressed in a timely manner. The foveal artery does not offer enough blood supply to supplement the loss of femoral artery flow. A varus fracture would be classified as a Garden III or IV due to the displacement and location of the injury [11].

\section{Clinical Presentation}

\section{Clinical overview}

Patients usually present with ambiguous pain in the upper leg that worsens with weight-bearing hip movements. Patients generally do not complain of swelling, loss of muscle tone, or weakness, but often refer to pain in the groin. Patients are at increased risk if they have a history of intensive training, are female athletes, suffer from coxa vara (deformity of the femoral head and neck 
with less than 120 degrees respective to femoral shaft), or experience insufficiency secondary to a myriad of causes (metabolic bone disease, chronic renal disease, endocrinopathy, smoking, infection, bone tumor at femoral neck, bisphosphonate therapy, post radiation therapy) [12]. Special attention is given to long term bisphosphonate therapy due to its unique presentations and unknown pathophysiology.

\section{Bisphosphonates}

Elderly patients may undergo antiresorptive therapy for severe osteopenia or osteoporosis. Studies have shown there are two rare known complications from prolonged therapy use: Osteonecrosis of the jaw and femoral fractures. This is especially true for patients on denosumab. The femoral fractures demonstrate a unique radiographic sign, cortical peaking, but also initially begins as a stress injury. There is small cortical disruption with osseous edema and periosteal reaction, which over time worsens into a complete fracture if untreated. Typical location of bisphosphonate stress fractures of the femur includes subtrochanteric and lateral cortex of femoral shaft. Some unique characteristics on imaging include lack of comminution, cortical thickening (peaking), and transverse orientation (Case 6). The pathophysiology for BP associated atypical femoral fractures (AFF) is not fully known, studies have shown BP therapy does not impact periosteal and endosteal callus formation. However, termination of BP has shown to decrease the incidence of AFF [13].

\section{Physical exam}

Pathologic findings are determined from a hop test, fulcrum test, or antalgic gait test. The hop test instructs the patient to hop on the affected leg. Complaints of pain indicates a positive finding. The fulcrum test is conducted by applying a downward force on the distal end of the femur and elevating the affected leg proximally while the patient is sitting. Complaints of pain indicate not only positive findings, but also alludes to a potential bone pathology. Antalgic gait test refers to the distinctive short stride patients have when one lower extremity is painful during weight bearing activity. Patients prefer to have limited weight bearing on the affected side, causing the opposite lower extremity to touch the ground much earlier than a normal gait. Although this is not a sensitive sign, this particular gait is a compensatory reaction to decrease pain [7].

\section{Types of Imaging}

\section{Radiograph}

Radiographic findings are negative during the first 6 weeks for patients presenting with stress injury. Multiple studies have found limited diagnostic value in radiographing stress fractures, with a reported sensitivity of $15-35 \%$ on initial imaging [14].
However, in some cases a faint radiolucent line may be detected, indicating a stress fracture. In many cases, subtle findings can be seen on plain radiographs, especially with varus and valgus impaction fractures but are unfortunately mislabeled as osteophyte formation or anatomical variation instead of stress fracture complication. The slight shift in the femoral head that occurs with stress fractures may be difficult to measure on plain radiograph due to increased noise from the pubis and dependency on patient orientation. Due to these difficulties, the fracture is often undetected until the patient presents with an ongoing periosteal or endosteal reaction. While radiographs may not provide significant diagnostic value to stress fractures themselves, they can reveal other underlying causes, especially in fractures due to metabolic/hormonal insufficiency or malignancies. Signs of osteoporosis, osteopenia, osteomalacia, and lytic lesions on radiograph can indicate a such an etiology and may warrant further examination for a possible stress fracture.

\section{Bone scintigraphy}

Triple phase technetium-99 m bisphosphate bone scintigraphy (BS) was previously the imaging tool of choice for suspected stress fractures. Increased localized remodeling and inflammation causes an increase in tracer uptake within 6-72 hours [14]. Generally, a positive sign on all three phases of BS (vascular, soft tissue, and bone) indicates an acute stress fracture. BS alone cannot exclude infection, tumor, or other inflammatory reactions from the differential diagnosis. Due to the test's low specificity (approximately 86\%), another imaging modality must be utilized for confirmation of stress reaction [15]. Other limitations of BS include exposure to ionizing radiation and the inability to visualize cortical disruption or change, which limits, follow up and progression.

\section{Computed tomography (CT)}

Computed tomography can detect subtle fracture lines not previously seen in plain radiographs. It has a high sensitivity for fractures making it an adequate modality for stress fracture imaging. CT imaging is limited in detection of soft tissue edema and osteoedema (18\% of cases), both of which are early markers of stress reaction and possible stress fracture [16]. However, there are some radiographic $\mathrm{CT}$ signs that can indicate stress related reaction or injury. This includes trabecular thickening secondary to increased stress and force distribution, cortical disruption (callus formation) or cortical thickening due to periosteal reaction.

Recently, dual energy CT (DECT) has shown to be an alternative to the identification of bone marrow edema. Differences in energy level is being used to hyperattenuate various tissue compositions allowing for increased diagnosis of radiographically occult fractures. Signs such 
as osteoedema can be readily seen in virtual noncalcium (VNCa) DECT. This can help facilitate diagnosis in patients in a timely manner and provide an alternative to MR imaging [17]. Consideration should be given to the radiation exposure, especially in pediatric patients, before using $\mathrm{CT}$ to diagnose femoral stress fractures.

\section{Magnetic resonance imaging (MRI)}

MRI has become the gold standard for evaluation of stress fractures, especially in the femoral head and neck. It has both high sensitivity and specificity for stress fracture and also provides positive imaging findings as early as onset of symptoms. Unlike bone scintigraphy, MRI can successfully narrow down the differential diagnosis among other etiologies with similar symptom presentation, such as iliopsoas tear, tendonitis, avascular necrosis, etc. [18]. The biggest advantage for MRI is the detection of endosteal and periosteal edema, which indicates increased osteoblastic/osteoclastic activity. Due to improved tissue contrast and lack of radiation, repeated imaging can also be done to evaluate and document fracture progression. MRI can also differentiate acute injuries ver- sus chronic stress injuries based on periosteal edema on T2 or STIR imaging with associated marrow edema in acute injury or callus formation from increased osteoblastic activity in chronic injury. Patients will have decreased signal on T1 with associated increased signal on STIR indicating acute injury. Stress fractures are seen as band-like areas of low signal on T1 weighted images in the intramedullary space and may continue to the cortex usually surrounded by an osteoedema [14]. Typical signs of stress injury in the femur are located in the subtrochanteric region with osseous changes of the cortical margin or subchondral marrow. Recent studies illustrate sequences such as diffusion weighted imaging (DWI) can be used to identify bone marrow edema as an adjunct to normal MRI findings. Other clinical signs on DWI include osseous and soft tissue edema [19].

\section{Femur imaging summary and protocol}

As a protocol, patients are generally screened with a plain radiograph to rule out fractures. In scenarios where no fractures are visualized, but one is suspected MRI is recommended. MRI has a positive

Table 2: Grading scale of stress fractures based on MRI findings. Allows for analysis of severity and appropriate treatment options.

\begin{tabular}{|l|l|l|l|}
\hline Grade & Frederickson Description & Type & Treatment \\
\hline $\mathbf{1}$ (Low) & $\begin{array}{l}\text { Mild to moderate periosteal edema on T2; normal } \\
\text { marrow on T2 and T1 }\end{array}$ & Compression & $\begin{array}{l}\text { Generally non-surgical unless > } \\
50 \%\end{array}$ \\
\hline $\mathbf{2}$ (Low) & $\begin{array}{l}\text { Moderate to severe periosteal edema on T2; marrow } \\
\text { edema on T2 but not T1 }\end{array}$ & Tensile & $\begin{array}{l}\text { Generally surgical, individually } \\
\text { based decision }\end{array}$ \\
\hline $\mathbf{3}$ (High) & $\begin{array}{l}\text { Moderate to severe periosteal edema on T2; marrow } \\
\text { edema on T2 and T1 }\end{array}$ & Displaced & $\begin{array}{l}\text { Surgical emergency for reduction } \\
\text { and fixation }\end{array}$ \\
\hline $\mathbf{4}$ (High) & $\begin{array}{l}\text { Moderate to severe periosteal edema on T2; marrow } \\
\text { edema on T2 and T1 with fracture line }\end{array}$ & Atypical tensile & Non-Surgical \\
\hline
\end{tabular}

\section{Example Cases}

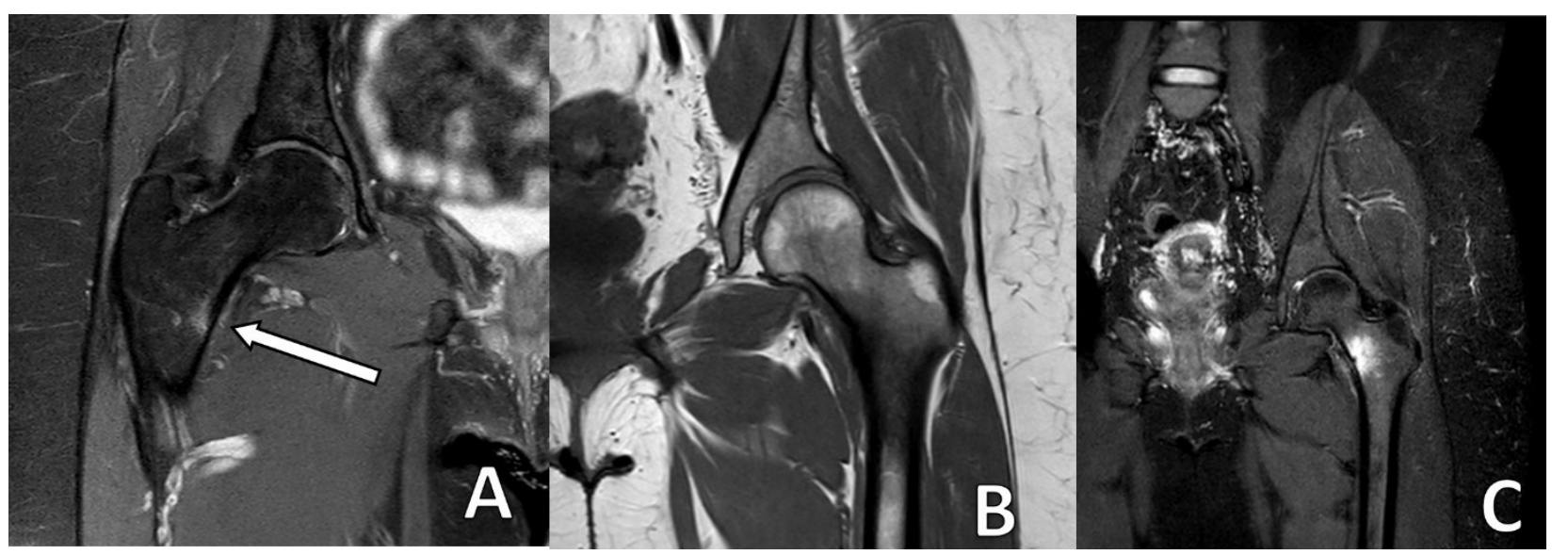

Patient 1: Images show a STIR and T1 with stress response and mild osteoedema on the compressive side of the medial intertrochanteric proximal right femur. There is no current evidence of fracture on far-left image; however, changes in the adjacent images indicate further progression could lead to stress fracture. White arrows show focal lesion with no surrounding soft tissue changes. Image B and C show common occurrence of medial sided stress fracture of the femoral neck. Changes are often seen on the compressive side of the femur either due to overexertion from the adductor muscle group or weakness of hip abductor muscle group. Compression-type stress fractures are characterized by a callus fracture pattern in the inferior aspect of the femoral neck with or without cortical disruption. These fractures are considered mechanically stable, and they are at a low risk of displacement [9]. 


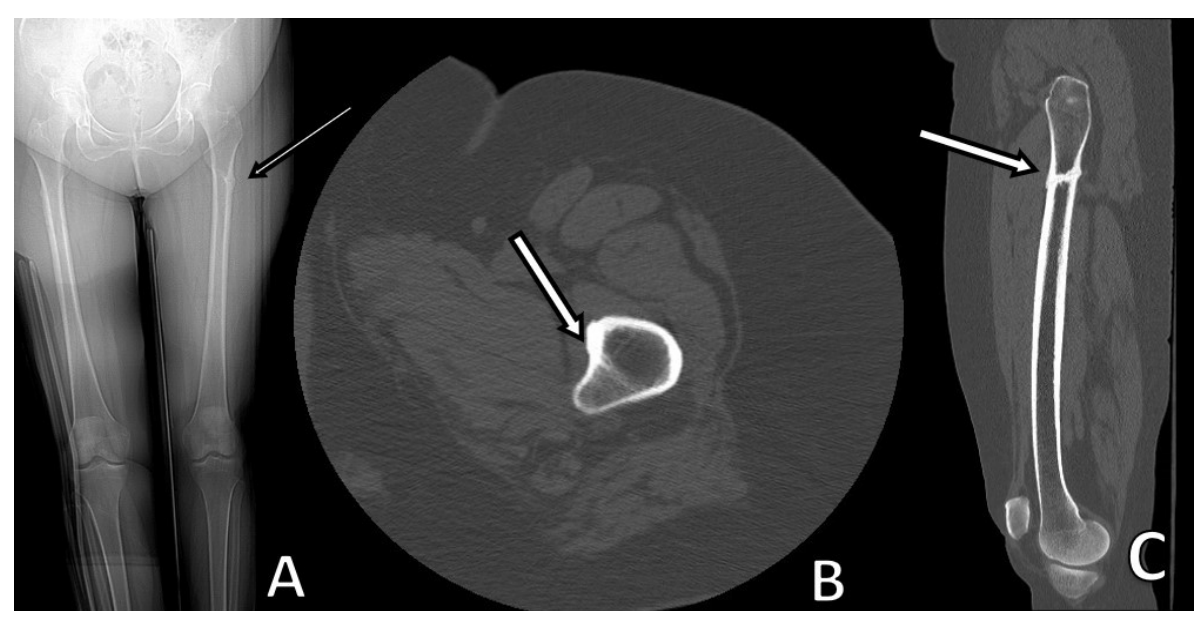

Patient 2 Image A: shows a subtle hypertrophic osteoblastic lesion on radiograph. Image B and C: CT imaging indicates a nondisplaced stress fracture involving of proximal left femoral shaft with evidence of attempt at healing.

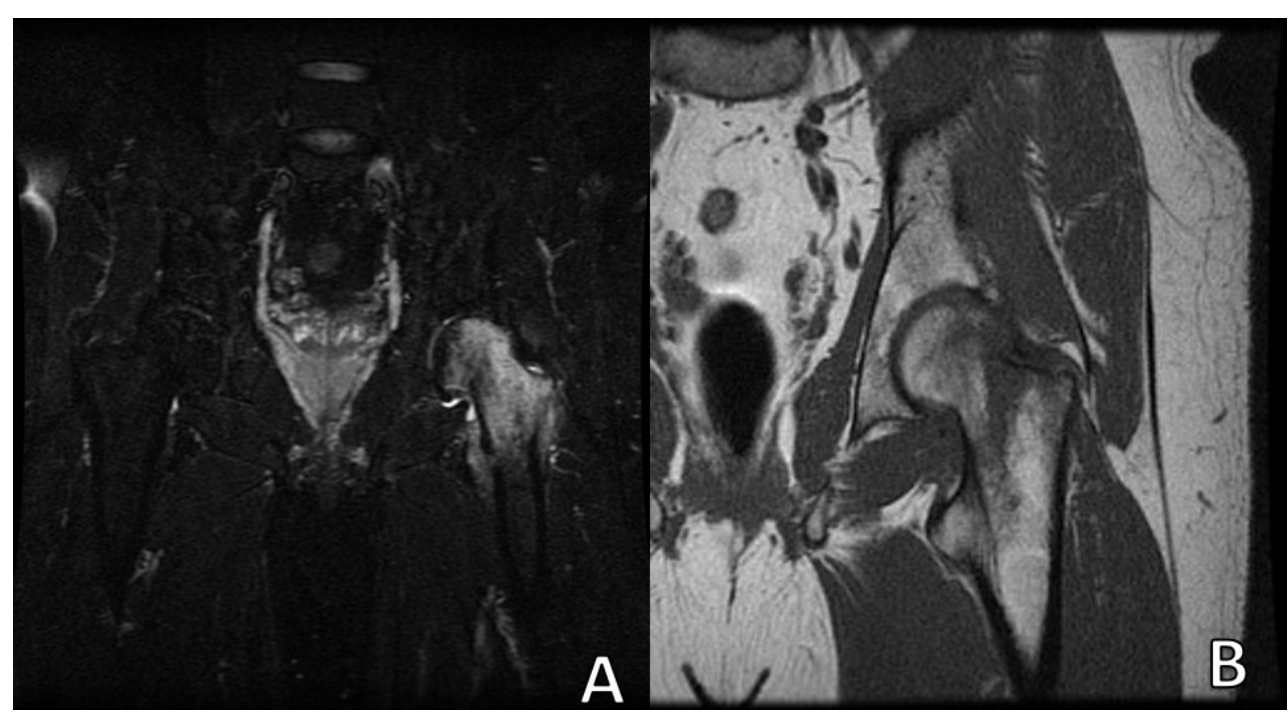

Patient 3 Image A: MRI T2 STIR indicating significant stress response of left proximal femur with diffuse edema. Image B: MRI T1 shows subchondral stress/insufficiency fracture of the superior left femoral neck with significant bone marrow edema in the left proximal femur. No evidence of subchondral collapse. MRI can help indicate the severity of damage and stress response versus traditional radiograph or CT.

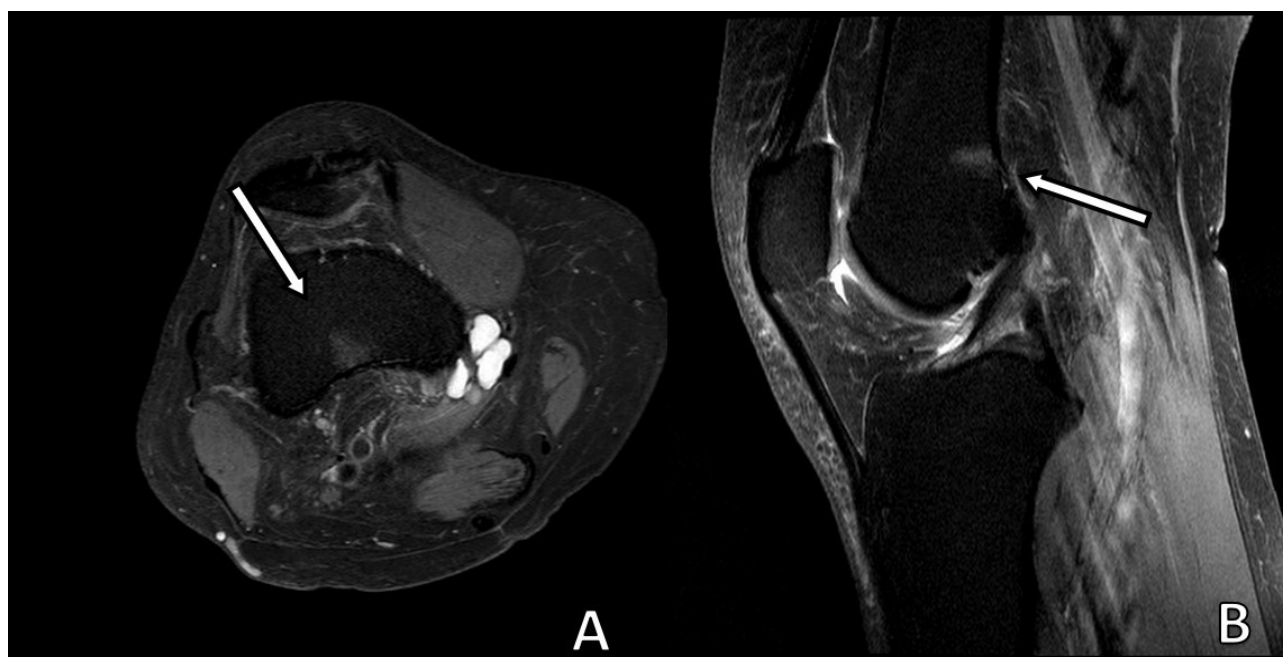

Patient 4 Image A and B: MR imaging shows focal linear region of marrow edema (T2 imaging) in the posterior distal femur, likely related to stress response. Subtle stress response can be difficult to identify on CT or radiographs. 


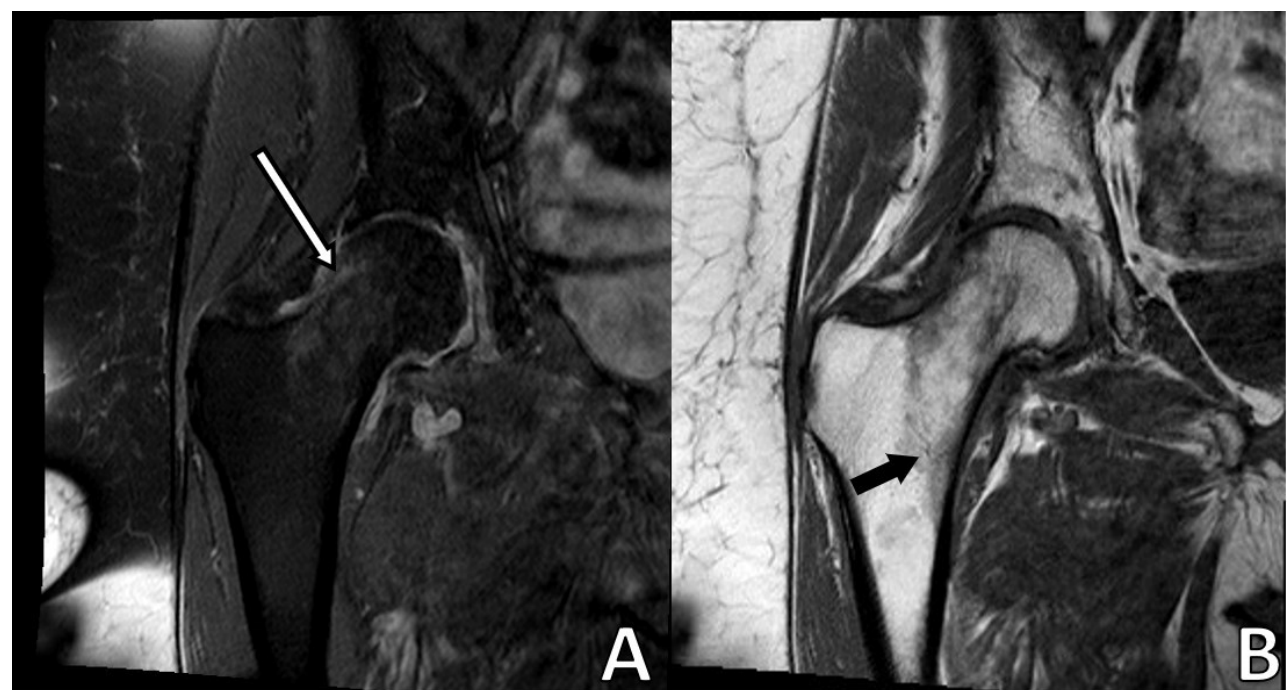

Patient 5 Image A: MRI T2 STIR shows an incomplete, nondisplaced fracture of the tensile surface of the right femoral neck. There is moderate bone marrow edema in the right femoral neck, greatest superiorly. White arrow: A thin linear, incomplete fracture extends superiorly towards the lateral aspect of the sub-capital femoral neck. Image B: MRI T1 with diffuse hypointense signaling at tensile surface extending into intertrochanteric region. Black Arrow: thin incomplete T1 hypointense fracture line extends inferiorly toward the proximal, anterior intertrochanteric region.

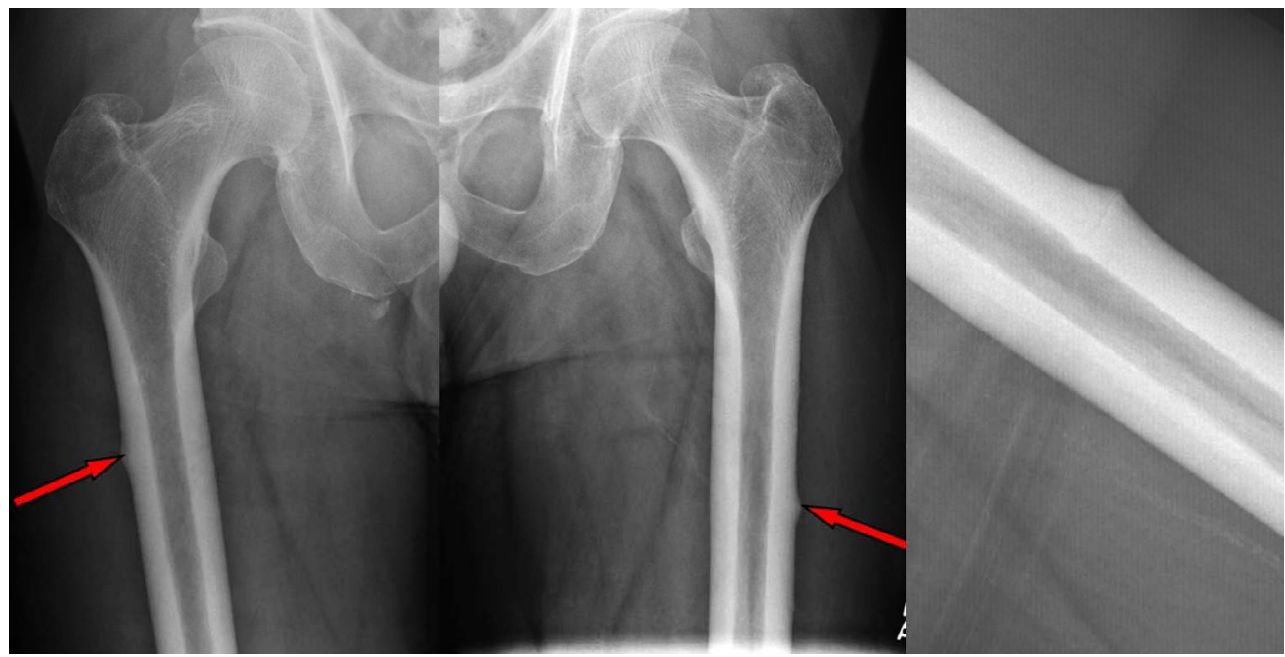

Patient 6: This patient has a history of long-term bisphosphonate therapy (more than 2 years). Bisphosphonates alter bone turnover and lead to insufficient matrix. Patients can develop stress injury from normal weight bearing activities red arrows indicate typical location of "cortical beaking" which can progress to a complete fracture.

predictive value equal to $100 \%$ for stress fractures of the hip [18]. Radiographic and MRI findings can include periosteal reaction and callus formation within the cortex (indicating older injury). There are multiple grading scales for stress fracture, one of the most common was created by Fredrickson which uses findings on T1 and T2 MRI imaging and associated edema (Table 2) $[1,20]$.

\section{Treatment}

Medical management can vary depending on the severity of the injury as well as the goals for each patient, the biggest decision in care being operative versus non-operative. Non-displaced stress fractures typically undergo non-operative management, with emphasis on rest for 3 - 16 weeks, dependent on the grade of stress fracture using the Frederickson crite- ria [21]. Often times the use of supportive crutches to minimize weight bearing stress is advised. However, this option is only feasible barring any displacement of the fracture, significant injury to adjacent structures, and/or disturbance to perfusion. If the patient suffers from any of these findings (including displacement), then surgical intervention may be warranted to preserve function of the lower extremity.

Patient who present with bisphosphonate associated stress injury or fracture, should stop the antiresorptive treatment. Often these patients are prophylactically treated operatively with an intramedullary nail [22].

\section{Hardware}

Depending on the location and grade of injury, various fixation methods are employed to allow for proper 
alignment and healing of the fracture. Depending on the degree of displacement, varus and valgus impaction fractures are generally fixated with long cannulated screws, securing the femoral head and aligning it to the appropriate angle (125-130 degrees) relative to the femoral shaft [4].

Metaphyseal stress fractures are generally treated non-operatively with restriction to weight bearing and activity. However; if in the presence of a displaced fracture, an intramedullary nail is typically indicated for appropriate fixation. If intramedullary nail is inappropriate, then a series of plates and screws can be used to align the femoral shaft.

\section{Conclusion}

Stress fractures are multifactorial. Their clinical history and physical examination can be ambiguous and often lead to a vague diagnosis. Patients with stress fracture of the femur can present with generalized groin pain, occasional swelling, worsening symptoms over time, and inability to bear weight for extended periods. Rarely is there a specific inciting traumatic event, but rather a gradual change in their health and activity level. The femur is comprised of a trabecular meshworks that is equipped to handle both intrinsic and extrinsic (tensile and compressive) forces. Overtime, the bone remodels to adequately distribute and absorb maximum forces on the femur. The etiology of stress fractures falls into two typical categories, fatigue or insufficiency fractures. Fatigue fractures are caused by an increase in weight bearing activity or stress on the femur leading to improper bone remodeling. Insufficiency fractures are caused by an underlying metabolic imbalance or deficiency that affects bone quality and modeling. There are two types of stress fractures, tensile and compressive. Generally, stress injury to the lateral aspect of the femur is associated with tensile forces. Lesions on the medial aspect of the femur and femoral neck are considered to be a compressive stress fracture. Clinical history can indicate underlying stress injury and may warrant a CT or MRI for further evaluation. Radiographic signs such as cortical disruption, peaking, periosteal edema, osseous edema, and adjacent soft tissue edema can indicate stress reaction or fracture. Using these radiological signs and the Frederickson criteria for MRI imaging of the femur, clinicians can deduce and grade the stress injury or fracture. Unrecognized stress injuries of the femur, especially the femoral neck, can lead to complications such as displacement and femoral head necrosis. Diagnosed stress fractures are medically managed with pain control and removal of the inciting event. In the cases where the femur is likely to be further compromised, hardware fixation can be performed.

All authors contributed to the manuscript in organizing and editing the review article.

\section{References}

1. Fredericson M, Un Jang K, Bergman G, Gold G (2004) Femoral diaphyseal stress fractures: Results of a systematic bone scan and magnetic resonance imaging evaluation in 25 runners. Physical Therapy in Sport 5: 188-193.

2. Frost HM (1994) Wolff's law and bone's structural adaptations to mechanical usage: An overview for clinicians. Angle Orthod 64: 175-188.

3. Tsubota K, Suzuki Y, Yamada T, Hojo M, Makinouchi A, et al. (2009) Computer simulation of trabecular remodeling in human proximal femur using large-scale voxel FE models: Approach to understanding wolff's law. J Biomech 42: 1088-1094.

4. Sheehan SE, Shyu JY, Weaver MJ, Sodickson AD, Khurana B, et al. (2015) Proximal femoral fractures: What the orthopedic surgeon wants to know. Radiographics 35: 15631584.

5. Gerber $H, V u T H$, Ryan AM, Kowalski J, Werb Z, et al. (1999) VEGF couples hypertrophic cartilage remodeling, ossification and angiogenesis during endochondral bone formation. Nat Med 5: 623-628.

6. Cooper KL (1994) Insufficiency stress fractures. Current Problems in Diagnostic Radiology 23: 31-68.

7. Defranco MJ, Recht M, Schils J, Parker RD (2006) Stress fractures of the femur in athletes. Clin Sports Med 25: 89103.

8. Devas M (1965) Stress fractures of the femoral neck. J Bone Joint Surg Br 47: 728-738.

9. Provencher MT, Baldwin AJ, Gorman JD, Gould MT, Shin AY (2004) Atypical tensile-sided femoral neck stress fractures: The value of magnetic resonance imaging. Am J Sports Med 32: 1528-1534.

10. Mears SC (2014) Classification and surgical approaches to hip fractures for nonsurgeons. Clin Geriatr Med 30: 229241.

11. Huang SY, Grimsrud CD, Provus J, Hararah M, Chandra M, et al. (2012) The impact of subtrochanteric fracture criteria on hip fracture classification. Osteoporos Int 23: 743-750.

12. Harris JD, Chahal J (2015) Femoral neck stress fractures. Operative Techniques in Sports Medicine 23: 241-247.

13. Shane E, Burr D, Abrahamsen B, Adler RA, Brown TD, et al. (2014) Atypical subtrochanteric and diaphyseal femoral fractures: Second report of a task force of the American society for bone and mineral research. J Bone Miner Res 29: 1-23.

14. Moran DS, Evans RK, Hadad E (2008) Imaging of lower extremity stress fracture injuries. Sports Medicine 38: 345356.

15. Berger FH, de Jonge MC, Maas M (2007) Stress fractures in the lower extremity. the importance of increasing awareness amongst radiologists. Eur J Radiol 62: 16-26.

16. Feydy A, Drapé J, Beret E, Sarazin L, Pessis E, et al. (1998) Longitudinal stress fractures of the tibia: Comparative study of CT and MR imaging. Eur Radiol 8: 598-602.

17. Ali IT, Wong WD, Liang T, Khosa F, Mian M, et al. (2018) Clinical utility of dual-energy CT analysis of bone marrow edema in acute wrist fractures. AJR Am J Roentgenol 210: 842-847.

18. Shin AY, Morin WD, Germany JD, Jones SB, Lapinsky AS (1996) The superiority of magnetic resonance imaging in differentiating the cause of hip pain in endurance athletes. Am J Sports Med 24: 168-176. 
19. Neubauer $H$, Evangelista L, Morbach $H$, Girschick $H$, Prelog M, et al. (2012) Diffusion-weighted MRI of bone marrow oedema, soft tissue oedema and synovitis in paediatric patients: Feasibility and initial experience. Pediatr Rheumatol Online J 10: 20.

20. Bencardino JT, Stone TJ, Roberts CC, Appel M, Bacce SJ, et al. (2017) ACR appropriateness criteria((R)) stress (fatigue/insufficiency) fracture, including sacrum, excluding other vertebrae. J Am Coll Radiol 14: S293-S306.

21. Caesar BC, Roberts SJ (2009) Stress fractures of the femoral diaphysis. Operative Techniques in Sports Medicine 17: 94-99.

22. Donnelly E, Saleh A, Unnanuntana A, Lane JM (2012) Atypical femoral fractures: Epidemiology, etiology, and patient management. Curr Opin Support Palliat Care 6: 348-354. 